\title{
LAMP assay coupled with CRISPR/Cas12a system for portable detection of African swine fever virus
}

\author{
Bo YANG ${ }^{1}$, zhengwang $\mathrm{shi}^{1}$, Yuan $\mathrm{Ma}^{1}$, Lijuan Wang ${ }^{1}$, Liyan $\mathrm{Cao}^{1}$, Juncong Luo ${ }^{1}$, Ying \\ Wang $^{1}$, Rui Song ${ }^{1}$, Yiyong yan ${ }^{2}$, kehu yuan ${ }^{2}$, Hong Tian ${ }^{1}$, and Haixue Zheng ${ }^{1}$ \\ ${ }^{1}$ State Key Laboratory of Veterinary Etiological Biology \\ ${ }^{2}$ Research and Development Department Shenzhen Bioeasy Biotechnology Co Ltd \\ Shenzhen Guangdong 518101 China
}

May 10, 2021

\begin{abstract}
African swine fever (ASF) is one of the most severe infectious diseases of pigs. In this study, a LAMP assay coupled with the CRISPR Cas12a system was established in one tube for the detection of the ASFV p72 gene. The single-strand DNAfluorophore-quencher (ssDNA-FQ) reporter and CRISPR-derived RNA (crRNAs) were screened and selected for the CRISPR detection system. In combination with LAMP amplification assay, the detection limit for the LAMP-CRISPR assay can reach 7 copies/ $\mu$ l of p72 gene per reaction. Furthermore, this method displays no cross-reactivity with other porcine DNA or RNA viruses. The performance of the LAMP-CRISPR assay was compared with real-time qPCR tests for clinical samples, a good consistency between the LAMP-CRISPR assay and real-time qPCR was observed. The method shed a light on the convenient, portable, low cost, highly sensitive and specific detection of ASFV, demonstrating a great application potential for monitoring on-site ASFV in the field.
\end{abstract}

\section{Introduction}

African swine fever (ASF) is a highly lethal contagious disease of swine caused by the African swine fever virus (ASFV). ASF affects both domestic and wild suids of all breeds and ages, with a high mortality rate of nearly 100\% (Parker et al., 1969; Anderson et al., 1998). Normally, ASF presents with high fever, cyanosis of the skin and severe hemorrhages in the lymph nodes. ASFV is a large and complex double-stranded DNA arbovirus that is the only member of the Asfarviridae family, Asfivirus genus(Alonso et al., 2018).

At present, there is no treatment or effective vaccine commercially available (Penrith and Vosloo, 2009), ASFV usually causes acute infection and it causes death before the production of protective antibody. Therefore, the early detection of ASFV plays an important role in the prevention and control of the disease. Both conventional and quantitative PCR are recommended by the World Organization for Animal Health (OIE) as the gold standard for the detection of the ASFV genome (Aguero et al., 2003; King et al., 2003; Aguero et al., 2004; Zsak et al., 2005). However, these methods require an expensive instruments and skilled operators, which limits the application of these methods for on-site situations. Isothermal amplification techniques, such as recombinase polymerase amplification (RPA) (Wang et al., 2017; Miao et al., 2019; Fan et al., 2020; Zhai et al., 2020), loop-mediated isothermal amplification (LAMP) (James et al., 2010; Mee et al., 2020; Wang et al., 2020a) and cross-priming amplification (CPA) (Fraczyk et al., 2016), have been successfully used to detect ASFV. Moreover, those isothermal amplification assays in combination with immunochromatographic strips have also been developed for application in the field. The main drawback of these techniques is the lack of high specificity and sensitivity, which limits their application in the detection of ASFV. 
Recently, nucleic acid detection techniques based on the clustered regularly interspaced short palindromic repeats (CRISPR)-associated endonucleases (CRISPR/Cas) systems have been developed (Chen et al., 2018; Gootenberg et al., 2018; Li et al., 2018). The detection relies on the cleavage preferences of Cas12 or Cas13 in a nonspecific way after binding to a specific target DNA or RNA via programmable guide RNAs. Combined with isothermal amplification RPA assay, the CRISPR system has been used for detecting ASFV(Bai et al., 2019; He et al., 2020; Li et al., 2020; Lu et al., 2020; Wang et al., 2020b; Wu et al., 2020; Ren et al., 2021). CRISPR/Cas-based diagnostic technology has been successfully applied to detect a variety of human viruses, such as Zika virus (ZIKV) (Gootenberg et al., 2018), Dengue virus (DENV) and human papillomavirus (HPV) (Tsou et al., 2019). However, the high cost of RPA assay limits its application in the field.

To improve the existing tools and to overcome the limitations for ASF diagnosis. Here, the low-cost LAMP amplification assay integrates with CRISPR Cas12a-based detection was developed. Compare with RPACRISPR based assays, LAMP-CRISPR uses less enzyme and less labor work, which is more efficient and time saving. This inexpensive, highly sensitive and specific, portable and visual method will be an alternative way for on-site ASFV detection, which might contribute to a timely monitoring and rapid strategy making for control of ASF.

\section{Materials and Methods}

\subsection{Biosafety Statement and Facility}

All experiments with live ASF viruses were conducted within the enhanced biosafety level 3 (P3) facilities at LVRI and were approved by the Ministry of Agriculture and Rural Affairs and the China National Accreditation Service for Conformity Assessment.

\subsection{Reagents and Instruments}

NEBuffer 2.1, EnGen Lba Cas12a (LbCas12a) were purchased from New England Biolabs (MA, United States). RNase inhibitor and ssDNA reporter were obtained from TAKARA (Tokyo, Japan). Fluorescence intensity was measured by QuantStudio 5 (Applied Biosystems, Massachusetts, USA). Five Cas12a crRNAs targeting the LAMP amplification of p72 were synthesized from GenePharma (Shanghai, China). The fluorescence signal of degraded ssDNA-FQ report was visualized with a UV light transilluminator (Tiangen, Beijing, China).

\subsection{Oligonucleotide primers for LAMP}

Oligonucleotide primers specific for ASFV were designed based on its p72 gene. Multiple sequences of p72 were aligned using MegAlign of the DNASTAR program (Version 7.1, DNASTAR, Madison, WI, USA). The most conserved region of the gene was subjected to design LAMP primers using the PrimerExplorer V4 software (http://primerexplorer.jp). The LAMP primers comprised two outer primers F3, B3 and two inner primers FIP, BIP. Five sets of primers were designed.

\subsection{Optimization and evaluation of the LAMP assays}

The LAMP reaction was conducted in a LAMP reaction mixture containing $12 \mu$ l of $2 \times$ LAMP buffer [40 $\mathrm{mM}$ Tris- $\mathrm{HCl}, \mathrm{pH} 8.8,20 \mathrm{mM} \mathrm{KCl}, 16 \mathrm{mM} \mathrm{MgSO}_{4}, 20 \mathrm{mM}\left(\mathrm{NH}_{4}\right)_{2} \mathrm{SO}_{4}, 0.2 \%$ Tween 20, $1.6 \mathrm{M}$ Betaine and $2.8 \mathrm{mM}$ of each dNTP], The outer primers and inner primers were optimized from $50 \mathrm{nM}$ to $600 \mathrm{nM} .1 .0 \mu \mathrm{l}$ Bst DNA polymerase (New England Biolabs, MA, USA), $2.0 \mu$ l of extracted DNA and $4 \mu \mathrm{l}$ of distilled water. The reaction mixture was performed in ABI QuantStudio 5. In addition, the LAMP reaction temperature was assessed by testing temperatures between $61^{\circ} \mathrm{C}$ and $67^{\circ} \mathrm{C}$.

\subsection{Evaluation of the sensitivity and fluorescence intensity of CRISPR Cas12a Detection}

CRISPR Cas12a detection assays contained 50nM LbCas12a, $100 \mathrm{nM}$ crRNA, $0.5 \mu$ RNase inhibitor, 2 $\mu l$ NEBuffer 2.1, FAM-BHQ1-labeled ssDNA reporter and various amount of dsDNA template p3xFLAGCMV-7.1-p72. The sample without dsDNA plasmid template was set as negative control. Reactions were 
incubated at a constant temperature of $37^{\circ} \mathrm{C}$ for $30 \mathrm{~min}$ and the fluorescent intensity was measured by $\mathrm{ABI}$ QuantStudio 5. In addition, fluorescence was observed with a UV transilluminator.

\subsection{LAMP-CRISPR fluorescence assay}

LAMP-CRISPR fluorescence assays include LAMP amplification of the dsDNA template and an optimized Cas12a cleavage assay. The LAMP reaction was added to the bottom of the PCR tube and Cas12a reaction was placed into the cap of the tube (Figure 1). Reactions were firstly incubated at optimized LAMP reaction temperature for $30 \mathrm{~min}$. Then, the PCR tube was centrifuged thoroughly to mix the LAMP reaction and Cas12a reaction. The mixed reaction was performed and the fluorescent intensity was measured using ABI. The fluorescence can be observed with a transilluminator.

The p3xFLAG-CMV-7.1-p72 dsDNA was serially diluted from $7 \times 10^{6}$ to $7 \times 10^{0}$ copies/ $\mu$. The sensitivity of the LAMP-CRISPR was analyzed with those of different dilutions of p72 dsDNA template. The fluorescent intensity was measured by ABI. To further determine the specificity of the developed LAMP-CRISPR assay, six porcine viruses including Classical Swine Fever Virus (CSFV), Foot-and-Mouth Disease Virus (FMDV), Senecavirus A (SVA), Porcine Circovirus 2 (PCV2), Porcine Epidemic Diarrhea Virus (PEDV) and Porcine Reproductive and Respiratory Syndrome (PRRSV) were tested.

\subsection{Comparison of LAMP-CRISPR with Taqman ${ }^{\circledR}$ real-time qPCR from clinical samples}

The Taqman real-time qPCR detection of the ASFV B646L (p72) gene was performed using a QuantStudio 5 system according to the procedure recommended from OIE described previously (King et al., 2003). The primers and Taqman probe were shown in Table 1. Briefly, $10 \mu \mathrm{l}$ of probe Master Mix (Takara, Dalian), 0.4 $\mu \mathrm{l}$ of primer F, $0.4 \mu \mathrm{l}$ of primer R, $3 \mu \mathrm{l}$ of DNA and $\mathrm{ddH}_{2} \mathrm{O}$. Reactions were conducted in a $25 \mu \mathrm{l}$ volume following the kit instructions. Reaction cycle parameters were set as denaturation at $95^{\circ} \mathrm{C}$ for 5 min, followed by 45 cycles of amplification, $95{ }^{\circ} \mathrm{C}$ for $15 \mathrm{~s}$ and $58^{\circ} \mathrm{C}$ for $60 \mathrm{~s}$. Pigs were infected with $10 \mathrm{HAD}_{50}$ ASFV (CN/GS/2018). Nasal and blood samples were collected at 1, 3, 5, 7, 9,11,13 and $15 \mathrm{dpi}$, tissue samples were also collected when pigs were dead. A total of 41 ASFV clinical samples were used to assess the performance of the LAMP-CRISPR. These materials comprised the nasal swab, spleen, liver, lung, submandibular lymph node and kidney samples from pigs collected in Lanzhou Veterinary Research Institute. Meanwhile, the real-time qPCR was also performed in parallel with DNA extracted from those samples.

\subsection{Statistical analysis}

Data were statistically processed by GraphPad Prism 5.0 (GraphPad Software, Inc., San Diego, CA, USA) for analysis of variance (ANOVA). The data are represented as means with standard deviation (SD) of three independent experiments. Results with p values of $<0.05$ were considered significant.

\section{Results}

\subsection{Establishing the fluorescent LAMP assay}

The fluorescent LAMP assay was used to screen the optimal primers from the designed 5 sets of primers, the concentration of primers and the reaction temperature. According to the application plot, the second set of primer has the highest efficiency and was selected to perform the LAMP assay (Supplementary Figure S1), the primers were shown in Table 1. The optimal concentration of outer primers (F3/B3) and inner primers were $100 \mathrm{nM}$ and $600 \mathrm{nM}$ (Supplementary Figure S2), respectively. The optimal LAMP reaction temperature was $63{ }^{\circ} \mathrm{C}$ (Supplementary Figure S3). In addition, the results also showed that the fluorescent LAMP assay can detect 7 copies $/ \mu l$ with the aid of ABI QuantStudio 5 .

\subsection{Quantitative direct detection of ASFV nucleic acid with CRISPR Cas12a}

We set out to develop a Cas12a-based direct detection assay for Viral DNA that would avoid the need for expensive instrument and enable point-of-care testing (Figure 1). To develop a sensitive and portable fluorescence detection system, 5 crRNAs were selected to optimize Cas12a activation performance. The results were shown in Figure 2A and 2B. According to the fluorescent intensity and the gradation of fluorescence, 
the crRNA 4 with the best active performance was selected to perform the CRISPR-Cas12a cleavage reporting reaction. Furthermore, the fluorescent intensity of ssDNA-FQ report 1 and ssDNA-FQ report 2 were compared under the $50 \mathrm{ng} / \mu \mathrm{l}$ and $5 \mathrm{ng} / \mu \mathrm{l}$ of DNA template. The results revealed that ssDNA-FQ reporter 2 had higher efficiency (Supplementary Figure S4). Moreover, $500 \mathrm{nM}$ of ssDNA-FQ reporter 2 was selected and used to perform our assay (Supplementary Figure S5).

\subsection{Sensitivity test of LAMP coupled with CRISPR Cas12a}

When combined the LAMP and CRISPR cleavage reaction, the sensitivity of the LAMP-CRISPR reaction was determined with a 10 -fold serial diluted template with a concentration of $7 \times 10^{6}$ copies $/ \mu l, 7 \times 10^{5}$ copies $/ \mu \mathrm{l}, 7 \times 10^{4}$ copies $/ \mu \mathrm{l}, 7 \times 10^{3}$ copies $/ \mu \mathrm{l}, 7 \times 10^{2}$ copies $/ \mu \mathrm{l}, 7 \times 10^{1} \mathrm{copies} / \mu \mathrm{l}$ and $7 \times 10^{0} \mathrm{copies} / \mu \mathrm{l}$ of the template, respectively. The results showed that the developed LAMP-CRISPR system can detect up to $7 \times 10^{1}$ copies $/ \mu$ l of the dsDNA template (Figure 3 ). To further improve the sensitivity of this assay, the proportion of the complex RNP formed by crRNA and Cas12a was further optimized with $7 \times 10^{1}$ copies $/ \mu$ l and $7 \times 10^{0}$ copies/ $\mu$ l of the template. The results indicated that when the ratio of crRNA to Cas12a is 1:1 (50nM:50nM), the LAMP-CRISPR are able to detect $7 \times 10^{0}$ copies/ $\mu$ l of the template in the reaction, which is the detection limit of this method. Moreover, 6 out of 10 target templates with 1 copies/ $\mu$ l can be detected by the LAMP-CRISPR system.

\subsection{Specificity test of LAMP coupled with CRISPR Cas12a}

To examine the specificity of LAMP-CRISPR detection of ASFV against other swine pathogens, including CSFV, FMDV, SVA, PCV2, PEDV and PRRSV, the tests were performed for detection of the abovementioned antigens. The results showed that all of these tested swine pathogens were negative, while only ASFV was positive (Figure 4), which demonstrated this method is highly specific for the detection of ASFV.

\subsection{Evaluating consistency between the LAMP-CRISPR assay and the commercial qPCR kit in porcine from clinical samples}

To evaluate the performance of the LAMP-CRISPR assay, 41 clinical samples including nasal swab, spleen, liver, lung, submandibular lymph node and kidney were assessed by both the LAMP-CRISPR and the commercial qPCR assay. As shown in Table 2, 5 out of 6 nasal swabs, 5 out of 7 spleens, 2 out of 7 livers, 6 out of 7 lungs, 5 out of 7 submandibular lymph nodes and 5 out of 7 kidney samples tested positive in both LAMP-CRISPR and qPCR assays, respectively (Figure 5). While only one more sample was positive in the liver when tested with the qPCR assay. The $C t$ value of that positive sample for qPCR is 36.12 , which indicated the number of virus copies from that sample is quite low. This result showed $96.6 \%$ consistency with these two assays, which supports LAMP-CRISPR can be regarded as a novel diagnostic assay for the detection of ASFV.

\section{Discussion}

The prevalence of ASF in a variety of countries causes a serious social and economic impact, which is limiting the trade of swine products and affecting food security. At present, molecular diagnostic techniques of ASF are mainly relying on two OIE-recommended conventional and real-time qPCR technique methods. Although these techniques have been widely validated and are useful tools for detecting of the disease, there is still lack of convenience because of the expensive instrument and professional operation system. In this study, a convenient, highly sensitive LAMP coupled with CRISPR-Cas12a assay was established for the rapid detection of ASFV.

LAMP assay has been used to detect ASFV with high sensitivity and efficiency (James et al., 2010; Wang et al., 2020a). The design of appropriate primers is one of the most important factors in optimizing the LAMP reaction. Four primers that recognize six distinct regions on the target are required (Notomi et al., 2000). In our study, five sets of primers were designed based on the conserved regions of the p72 gene, which is the most essential structural component of the virion, accounting for $31 \%-33 \%$ of the total mass of the virion (Carrascosa et al., 1984; Garcia-Escudero et al., 1998). According to the amplification plot of those primers, finally, one set of primers was selected to establish the LAMP assay. Moreover, analytical 
sensitivity indicated that the LAMP can detect 7 copies/ $\mu$ l of DNA template at $63^{\circ} \mathrm{C}$ for $30 \mathrm{~min}$. Although LAMP assay has high analytical sensitivity, cross-contamination LAMP product into pre-reaction mixtures can readily cause false-positive results. Thus, combining with the CRISPR system is effective to avoid the false-positive cases.

The fluorescence reporter is quite important for visualizing nucleic acids in the CRISPR Cas12a reaction. In this study, two FAM modified ssDNA-FQ reporters were tested. The results revealed that different ssDNA-FQ has a prodigious difference for visualization. Therefore, the most effective ssDNA-FQ reporter was selected in our study. More new types will be used to compare the effectiveness in the near future. Five crRNAs were designed to target ASFV p72, and one of which exhibited the highest activity based on the fluorescent signal. Parinaz et al have demonstrated that combinations of crRNA can increase the sensitivity of Cas13a detection by activating more Cas13a per target RNA (Fozouni et al., 2020). Furthermore, the use of multiple crRNAs that target different parts of the gene also safeguards against a potential loss of detection because of naturally occurring viral mutations. Future work will entail a combination of crRNAs to improve the sensitivity of nucleic acid detection through enhanced crRNA activity.

Previously, RPA coupled with CRISPR system has been established for on-site viral detection owing to the similar optimal reaction temperature between two steps. However, the cost of the RPA reaction is too high to be applied in the field. Therefore, in our study, LAMP combined with CRISPR Cas12a was developed at a single test tube. The LAMP reaction reagents can be first put in the bottom of the tube, the CRISPR reaction reagents remain stable within the cap of the tube. When the LAMP reaction is completed, the CRISPR reagents can be spun down into the tube for detection of ASFV. At this moment, it is important to notice that the temperature control of the test tube is essential, because the Cas12a can be inactivated under high temperature. In the near future, the annular tube will be used to contain LAMP and CRISPR reactions with different optimal temperatures.

The sensitivity of the LAMP-CRISPR method has demonstrated its capability of detecting ASFV. Cas12abased nucleic acid fluorescence reporting system reached a sensitivity level of $7 \times 10^{8}$ copies/ $\mu$ without amplification of DNA targets. In combination with LAMP amplification, the LAMP-CRISPR assay detected the DNA target at a sensitivity level of 7 copies/ $\mu$ l. Moreover, when diluted the DNA target as 1 copies/ $\mu l$, 6 out of 10 can be detected, which demonstrated its high sensitivity. In addition, the crude RNA extraction methods (e.g. by using high temperature or lysis buffer to release nucleic acid) that could be performed in the field has been tested. Indeed, the sensitivity of this method can be slightly decreased. The impact is not that significant, but a more appropriate and optimized extraction methods is necessary to developed for the on-site sample detection in the future. Furthermore, the results of the clinical samples test illustrated that the LAMP-CRISPR assay not only requires less time than real-time qPCR but also simplifies the detection process.

In conclusion, LAMP-CRISPR was established and used for the rapid, low-cost, sensitive, specific and portable detection of ASFV. LAMP-CRISPR has great potential for on-site ASFV detection, which could be an effective way for timely monitoring of ASFV to prevent the occurrence and spread of ASFV at an early stage.

\section{Competing interests}

The authors declare that they have no conflicts of interest.

\section{Ethical statements}

All animal experimental procedures have been reviewed and approved by the Animal Care and Use Committee of Lanzhou Veterinary Research Institute of the Chinese Academy of Agricultural Sciences (approval ID: SYXK(Gan) 2015-0003).

\section{Author's contribution}

BY performed the experiments, analyzed the data and drafted the manuscript. ZW, MY, LJ, WY and SR 
assisted in performing the experiment. $\mathrm{YY}$ and $\mathrm{KH}$ participated in writing the manuscript. TH and $\mathrm{HX}$ conceived and designed the study and helped in writing the manuscript. All authors read and approved the final manuscript.

\section{Acknowledgement}

The authors would like to thank Xiao Yang and Juan Wei for their excellent technical assistance. This work was supported by the National Key Research and Development Project (2018YFC0840400), the Gansu major science and technology project (20ZD7NA006), the Collaborative Innovation Project of Chinese Academy of Agricultural Sciences (CAAS-ZDRW202006), the Guangdong Innovative and Entrepreneurial Research Team Program (No. 2014ZT05S123) and the Shenzhen Science and Technology Innovation Commission (JSGG20180508152235868).

\section{Figure Legends}

Figure 1. Schematic diagram of the LAMP amplification assay coupled with CRISPR system. The pictures were captured under blue and UV lights by a smartphone camera or gel imaging system.

Figure 2. The efficiency of CRISPR/Cas12a trans-cleavage system induced by ASFV specific crRNAs. The fluorescent signal was collected induced by different crRNAs with ABI QuantStudio 5 (A) and visualized by gel imaging system using UV light (B).

Figure 3. Comparison of the detection limit between CRISPR/Cas12a system and LAMP-CRISPR assay. The fluorescent signals from a series of 10-fold dilutions of dsDNA template p3xFLAG-CMV-7.1-p72 plasmid.

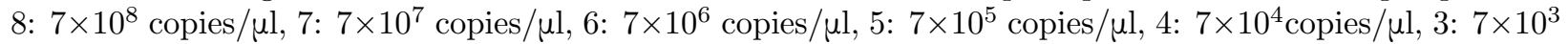

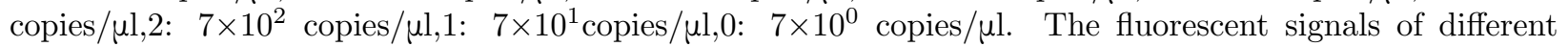
dilutions were calculated by ABI QuantStudio 5 (A and B) and visualized by gel imaging system using UV light (C and D) after performed with CRISPR/Cas12a system reaction and LAMP-CRISPR assay.

Figure 4. Specificity of LAMP-CRISPR assay for the detection of ASFV. The fluorescent signals were calculated by ABI QuantStudio 5 (A) and visualized by gel imaging system using UV light (B) with DNA viruses ASFV and PCV2, cDNA of CSFV, FMDV, SVA, PEDV and PRRSV by performing LAMP-CRISPR assay.

Figure 5. Detection of ASFV in swine samples. Samples from nasal swab (A and a), spleen (B and b), liver ( $\mathrm{C}$ and $\mathrm{c}$ ), lung ( $\mathrm{D}$ and $\mathrm{d})$, submandibular lymph node ( $\mathrm{E}$ and e) and kidney ( $\mathrm{F}$ and $\mathrm{f}$ ) were tested by LAMP-CRISPR assay, respectively, and the fluorescent signals were calculated by ABI QuantStudio 5 and visualized by gel imaging system using UV light. Besides, S5 from nasal swabs was not available, which was shown in empty tube from the picture a.

\section{Supplemental Figures}

Supplemental Figure S1. Application plot of fluorescent LAMP assay with 5 set of primers.

Supplemental Figure S2. Optimization of primer concentration with the fluorescent LAMP assay. The concentration ratio of outer primer and inner primer with $50 \mathrm{nM}: 200 \mathrm{nM}, 50 \mathrm{nM}: 400 \mathrm{nM}, 50 \mathrm{nM}: 600 \mathrm{nM}$, $100 \mathrm{nM}: 200 \mathrm{nM}, 100 \mathrm{nM}: 400 \mathrm{nM}, 100 \mathrm{nM}: 600 \mathrm{nM}, 200 \mathrm{nM}: 400 \mathrm{nM}$ and $200 \mathrm{nM}: 600 \mathrm{nM}$ was used to perform the fluorescent LAMP assay, respectively.

Supplemental Figure S3. Optimization of the fluorescent LAMP reaction temperature. The LAMP assay was performed under $61^{\circ} \mathrm{C}, 62^{\circ} \mathrm{C}, 63^{\circ} \mathrm{C}, 64^{\circ} \mathrm{C}, 65^{\circ} \mathrm{C}, 66^{\circ} \mathrm{C}$ and $67^{\circ} \mathrm{C}$ reaction temperature, respectively.

Supplemental Figure S4. Optimization of crRNA and ssDNA-FQ reporter for CRISPR/cas12a cleavage report system. CRSIPR/Cas12a reaction activity was compared between ssDNA-FQ reporter 1 and ssDNAFQ reporter 2 by using three different crRNA sets (crRNA 3, crRNA 4 and crRNA 5) under two different concentration of dsDNA template $(5 \mathrm{ng} / \mu \mathrm{l}$ and $50 \mathrm{ng} / \mu \mathrm{l})$. 
Supplementary Figure S5 . Optimization of ssDNA-FQ reporter concentration. The concentration of $100 \mathrm{nM}, 200 \mathrm{nM}, 300 \mathrm{nM}, 400 \mathrm{nM}$ and $500 \mathrm{nM}$ ssDNA-FQ was used to perform the CRISPR system assay, respectively. Amplification plot of CRISPR detection assay (A) and visualized by using UV light (B) with different concentration of ssDNA-FQ reporter.

\section{References}

Aguero, M., Fernandez, J., Romero, L., Sanchez Mascaraque, C., Arias, M., Sanchez-Vizcaino, J.M., 2003. Highly sensitive PCR assay for routine diagnosis of African swine fever virus in clinical samples. J Clin Microbiol 41, 4431-4434.

Aguero, M., Fernandez, J., Romero, L.J., Zamora, M.J., Sanchez, C., Belak, S., Arias, M., Sanchez-Vizcaino, J.M., 2004. A highly sensitive and specific gel-based multiplex RT-PCR assay for the simultaneous and differential diagnosis of African swine fever and Classical swine fever in clinical samples. Vet Res 35, 551563.

Alonso, C., Borca, M., Dixon, L., Revilla, Y., Rodriguez, F., Escribano, J.M., Ictv Report, C., 2018. ICTV Virus Taxonomy Profile: Asfarviridae. J Gen Virol 99, 613-614.

Anderson, E.C., Hutchings, G.H., Mukarati, N., Wilkinson, P.J., 1998. African swine fever virus infection of the bushpig (Potamochoerus porcus) and its significance in the epidemiology of the disease. Vet Microbiol $62,1-15$.

Bai, J., Lin, H., Li, H., Zhou, Y., Liu, J., Zhong, G., Wu, L., Jiang, W., Du, H., Yang, J., Xie, Q., Huang, L., 2019. Cas12a-Based On-Site and Rapid Nucleic Acid Detection of African Swine Fever. Front Microbiol $10,2830$.

Carrascosa, J.L., Carazo, J.M., Carrascosa, A.L., Garcia, N., Santisteban, A., Vinuela, E., 1984. General morphology and capsid fine structure of African swine fever virus particles. Virology 132, 160-172.

Chen, J.S., Ma, E., Harrington, L.B., Da Costa, M., Tian, X., Palefsky, J.M., Doudna, J.A., 2018. CRISPRCas12a target binding unleashes indiscriminate single-stranded DNase activity. Science 360, 436-439.

Fan, X., Li, L., Zhao, Y., Liu, Y., Liu, C., Wang, Q., Dong, Y., Wang, S., Chi, T., Song, F., Sun, C., Wang, Y., Ha, D., Zhao, Y., Bao, J., Wu, X., Wang, Z., 2020. Clinical Validation of Two Recombinase-Based Isothermal Amplification Assays (RPA/RAA) for the Rapid Detection of African Swine Fever Virus. Front Microbiol 11, 1696.

Fozouni, P., Son, S., Diaz de Leon Derby, M., Knott, G.J., Gray, C.N., D'Ambrosio, M.V., Zhao, C., Switz, N.A., Kumar, G.R., Stephens, S.I., Boehm, D., Tsou, C.L., Shu, J., Bhuiya, A., Armstrong, M., Harris, A.R., Chen, P.Y., Osterloh, J.M., Meyer-Franke, A., Joehnk, B., Walcott, K., Sil, A., Langelier, C., Pollard, K.S., Crawford, E.D., Puschnik, A.S., Phelps, M., Kistler, A., DeRisi, J.L., Doudna, J.A., Fletcher, D.A., Ott, M., 2020. Amplification-free detection of SARS-CoV-2 with CRISPR-Cas13a and mobile phone microscopy. Cell.

Fraczyk, M., Wozniakowski, G., Kowalczyk, A., Niemczuk, K., Pejsak, Z., 2016. Development of crosspriming amplification for direct detection of the African Swine Fever Virus, in pig and wild boar blood and sera samples. Lett Appl Microbiol 62, 386-391.

Garcia-Escudero, R., Andres, G., Almazan, F., Vinuela, E., 1998. Inducible gene expression from African swine fever virus recombinants: analysis of the major capsid protein p72. J Virol 72, 3185-3195.

Gootenberg, J.S., Abudayyeh, O.O., Kellner, M.J., Joung, J., Collins, J.J., Zhang, F., 2018. Multiplexed and portable nucleic acid detection platform with Cas13, Cas12a, and Csm6. Science 360, 439-444.

He, Q., Yu, D., Bao, M., Korensky, G., Chen, J., Shin, M., Kim, J., Park, M., Qin, P., Du, K., 2020. High-throughput and all-solution phase African Swine Fever Virus (ASFV) detection using CRISPR-Cas12a and fluorescence based point-of-care system. Biosens Bioelectron 154, 112068. 
James, H.E., Ebert, K., McGonigle, R., Reid, S.M., Boonham, N., Tomlinson, J.A., Hutchings, G.H., Denyer, M., Oura, C.A., Dukes, J.P., King, D.P., 2010. Detection of African swine fever virus by loop-mediated isothermal amplification. J Virol Methods 164, 68-74.

King, D.P., Reid, S.M., Hutchings, G.H., Grierson, S.S., Wilkinson, P.J., Dixon, L.K., Bastos, A.D., Drew, T.W., 2003. Development of a TaqMan PCR assay with internal amplification control for the detection of African swine fever virus. J Virol Methods 107, 53-61.

Li, S.Y., Cheng, Q.X., Liu, J.K., Nie, X.Q., Zhao, G.P., Wang, J., 2018. CRISPR-Cas12a has both cis- and trans-cleavage activities on single-stranded DNA. Cell Res 28, 491-493.

Li, Z., Wei, J., Di, D., Wang, X., Li, C., Li, B., Qiu, Y., Liu, K., Gu, F., Tong, M., Wang, S., Wu, X., Ma, Z., 2020. Rapid and accurate detection of African swine fever virus by DNA endonuclease-targeted CRISPR trans reporter assay. Acta Biochim Biophys Sin (Shanghai) 52, 1413-1419.

Lu, S., Li, F., Chen, Q., Wu, J., Duan, J., Lei, X., Zhang, Y., Zhao, D., Bu, Z., Yin, H., 2020. Rapid detection of African swine fever virus using Cas12a-based portable paper diagnostics. Cell Discov 6, 18.

Mee, P.T., Wong, S., O'Riley, K.J., da Conceicao, F., Bendita da Costa Jong, J., Phillips, D.E., Rodoni, B.C., Rawlin, G.T., Lynch, S.E., 2020. Field Verification of an African Swine Fever Virus Loop-Mediated Isothermal Amplification (LAMP) Assay During an Outbreak in Timor-Leste. Viruses 12.

Miao, F., Zhang, J., Li, N., Chen, T., Wang, L., Zhang, F., Mi, L., Zhang, J., Wang, S., Wang, Y., Zhou, X., Zhang, Y., Li, M., Zhang, S., Hu, R., 2019. Rapid and Sensitive Recombinase Polymerase Amplification Combined With Lateral Flow Strip for Detecting African Swine Fever Virus. Front Microbiol 10, 1004.

Notomi, T., Okayama, H., Masubuchi, H., Yonekawa, T., Watanabe, K., Amino, N., Hase, T., 2000. Loopmediated isothermal amplification of DNA. Nucleic Acids Res 28, E63.

Parker, J., Plowright, W., Pierce, M.A., 1969. The epizootiology of African swine fever in Africa. Vet Rec 85, 668-674.

Penrith, M.L., Vosloo, W., 2009. Review of African swine fever: transmission, spread and control. J S Afr Vet Assoc 80, 58-62.

Ren, M., Mei, H., Zhou, M., Fu, Z.F., Han, H., Bi, D., Peng, F., Zhao, L., 2021. Development of A SuperSensitive Diagnostic Method for African Swine Fever Using CRISPR Techniques. Virol Sin 36, 220-230.

Tsou, J.H., Leng, Q., Jiang, F., 2019. A CRISPR Test for Detection of Circulating Nuclei Acids. Transl Oncol 12, 1566-1573.

Wang, D., Yu, J., Wang, Y., Zhang, M., Li, P., Liu, M., Liu, Y., 2020a. Development of a real-time loopmediated isothermal amplification (LAMP) assay and visual LAMP assay for detection of African swine fever virus (ASFV). J Virol Methods 276, 113775.

Wang, J., Wang, J., Geng, Y., Yuan, W., 2017. A recombinase polymerase amplification-based assay for rapid detection of African swine fever virus. Can J Vet Res 81, 308-312.

Wang, X., Ji, P., Fan, H., Dang, L., Wan, W., Liu, S., Li, Y., Yu, W., Li, X., Ma, X., Ma, X., Zhao, Q., Huang, X., Liao, M., 2020b. CRISPR/Cas12a technology combined with immunochromatographic strips for portable detection of African swine fever virus. Commun Biol 3, 62.

Wu, J., Mukama, O., Wu, W., Li, Z., Habimana, J.D., Zhang, Y., Zeng, R., Nie, C., Zeng, L., 2020. A CRISPR/Cas12a Based Universal Lateral Flow Biosensor for the Sensitive and Specific Detection of African Swine-Fever Viruses in Whole Blood. Biosensors (Basel) 10.

Zhai, Y., Ma, P., Fu, X., Zhang, L., Cui, P., Li, H., Yan, W., Wang, H., Yang, X., 2020. A recombinase polymerase amplification combined with lateral flow dipstick for rapid and specific detection of African swine fever virus. J Virol Methods 285, 113885. 
Zsak, L., Borca, M.V., Risatti, G.R., Zsak, A., French, R.A., Lu, Z., Kutish, G.F., Neilan, J.G., Callahan, J.D., Nelson, W.M., Rock, D.L., 2005. Preclinical diagnosis of African swine fever in contact-exposed swine by a real-time PCR assay. J Clin Microbiol 43, 112-119.

\section{Hosted file}

Revised supplemental figures S1-S5.pdf available at https://authorea.com/users/394813/ articles/521431-lamp-assay-coupled-with-crispr-cas12a-system-for-portable-detectionof-african-swine-fever-virus

\section{Hosted file}

Revised figures.pdf available at https://authorea.com/users/394813/articles/521431-lampassay-coupled-with-crispr-cas12a-system-for-portable-detection-of-african-swine-fevervirus

\section{Hosted file}

Supplementary table 1.pdf available at https://authorea.com/users/394813/articles/521431lamp-assay-coupled-with-crispr-cas12a-system-for-portable-detection-of-african-swinefever-virus

\section{Hosted file}

Revised tables.pdf available at https://authorea.com/users/394813/articles/521431-lamp-assaycoupled-with-crispr-cas12a-system-for-portable-detection-of-african-swine-fever-virus 